\title{
Narrativa
}

\section{Immaginare il futuro: le narrazioni distopiche nell'Italia del terzo millennio}

\section{Aleksandra Pogonska-BaranowsKa}

\section{(2) OpenEdition}

\section{Journals}

Edizione digitale

URL: https://journals.openedition.org/narrativa/368

DOI: $10.4000 /$ narrativa.368

ISSN: 2804-1224

\section{Editore}

Presses universitaires de Paris Nanterre

\section{Edizione cartacea}

Data di pubblicazione: 1 décembre 2019

Paginazione: 157-167

ISBN: 978-2-84016-350-3

ISSN: $1166-3243$

Notizia bibliografica digitale

Aleksandra Pogonska-BaranowsKa, «Immaginare il futuro: le narrazioni distopiche nell'Italia del terzo millennio», Narrativa [Online], 41 | 2019, online dal 01 septembre 2021, consultato il 08 décembre 2021 URL: http://journals.openedition.org/narrativa/368 ; DOI: https://doi.org/10.4000/narrativa.368

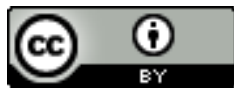

Narrativa est mise à disposition selon les termes de la Licence Creative Commons Attribution 4.0 International. 


\title{
Immaginare il futuro: le narrazioni distopiche nell'Italia del terzo millennio
}

\begin{abstract}
Riassunto
La protezione del genere umano dai rischi connessi al suo (smisurato) potere risulta uno degli obiettivi fondamentali dei progetti dell'umanità nel xxI secolo, diventando così un motivo ricorrente della letteratura contemporanea. Il saggio propone una mappatura della variante distopica del romanzo italiano, tornata in auge all'inizio del terzo millennio. L'analisi delle tendenze tematiche e ideologiche delle narrazioni distopiche è preceduta da una premessa teorico-tassonomica e dalla presentazione delle attuali tipologie distopiche.
\end{abstract}

\section{RÉSUMÉ}

La protection du genre humain par rapport aux risques liés à son pouvoir (démesuré) représente l'un des objectifs fondamentaux des projets de l'humanité au xxi siècle, en devenant ainsi un motif récurrent de la littérature contemporaine. Cet article propose une cartographie de la variante dystopique du roman italien, revenue sur le devant de la scène au début de ce millénaire. L'analyse des tendances thématiques et idéologiques des narrations dystopiques est précédée d'une introduction théorique-taxonomique et de la présentation des typologies dystopiques actuelles.

\footnotetext{
ll'inizio del terzo millennio, l'umanità prova a risvegliarsi dall'incubo del secolo appena trascorso, afflitto da numerose disfunzioni e terribili patologie che hanno rivelato una natura profondamente apocalittica. Accompagnato dalla stupefacente constatazione che da qualche decennio è riuscito a trasformare carestie, pestilenze e guerre da incomprensibili e incontrollabili forze della natura in sfide che possono essere affrontate, assicurando livelli di prosperità, salute e armonia quasi utopici, il genere umano sembra poter sollevare lo sguardo e volgerlo verso nuovi orizzonti. Spinto dai recenti trionfi,
} 
coltiva l'ambizione suprema di elevare gli umani al rango di divinità, di trasformare Homo sapiens in Homo Deus ${ }^{1}$.

Gli ultimi vent'anni, orientati allo sviluppo economico e segnati dall'enorme balzo in avanti della tecnologia, invece di dar luogo a manifestazioni di ottimismo sfrenato, hanno fornito motivi giustificati di timori, mostrati e denunciati da diversi rappresentanti della letteratura contemporanea. Il potenziale distruttivo dell'enorme arsenale tecnico di cui oggi disponiamo sta diventando sempre più evidente. L'appello alla ragionevolezza, alla morale, alla prudenza nella sperimentazione di tecniche manipolatrici della natura e dell'uomo che ne è parte, rivela la pericolosità dei processi in atto. Sembra che l'uomo, che non ha mai avuto motivo di vivere scevro da trepidazione in questo mondo, non sia oggi esente dal timore, innanzitutto di se stesso, delle proprie azioni e delle relative conseguenze.

È proprio negli ultimissimi anni che le opere di matrice apocalittica e distopica mostrano un cambiamento radicale. Non più guerre stellari ma global warming $\mathrm{e}$ plasticsoup nell'oceano colpiscono l'immaginazione degli scrittori contemporanei. E accanto alle emergenze da cambiamenti climatici e inquinamento, nanoparticelle, cibo e acqua contaminati si temono più di moti tettonici, eruzioni vulcaniche o perfino minacce nucleari; c'è una crescente preoccupazione per la la tensione o libridazione tra physsis e téchne, e in parallelo tra uomo e macchina, per le nep news e la manipolazione dei mass media, per le sfide e le minacce legate al crescente problema della migrazione. La protezione del genere umano dai rischi connessi al suo proprio potere e alla sua audacia diventa uno degli obiettivi fondamentali della lista dei progetti dell'umanità nel XxI secolo, diventando uno dei motivi più ricorrenti delle visioni distopiche nella letteratura del terzo millennio, chiamato spesso una nuova "epoca d'oro della narrativa distopica"

1. Harrari Yuval Noah, Homo Deus. Breve storia del futuro, Milano, Bompiani, 2017, p. 31.

2. Secondo Carlo Bordoni, il tempo dell'età d'oro delle distopie ha coinciso con $\mathrm{i}$ grandi totalitarismi che hanno afflitto l'Europa nel secolo scorso. Dal Tallone di ferro di Jack London a 1984 di George Orwell, il xx secolo è stato un fiorire di previsioni funeste sul futuro dell'individuo sopraffatto dalla società massificata e oppresso da regimi antidemocratici. Bordoni parla di un fenomeno che "non ha uguali nella storia passata". Nessun secolo precedente ha manifestato in letteratura con tanta determinazione il proprio pessimismo per il domani (Bordoni Carlo, "Della distopia o del pessimismo futuro", IF, Anno III, n. 7, luglio 2011, p. 3).

3. Lepore Jill, "A Golden Age for Dystopian Fiction: What to make of our new literature of radical pessimism”, The New Yorker, 29 maggio 2017, https:/ /www.newyorker. $\mathrm{com} /$ magazine/2017/06/05/a-golden-age-for-dystopian-fiction 
Prima di analizzare le raffigurazioni letterarie italiane dell'utopia e della distopia $^{4}$ (detta anche utopia negativa ${ }^{5}$ ), occorre fare alcune precisazioni preliminari di ordine tassonomico e sottolineare un nesso tra le due nozioni. L'una, intesa a sostenere ideologicamente e psicologicamente la volontà di conseguire lo scopo prefissato di immaginare un mondo perfetto, diventa un'espressione di desiderio, ottimismo e speranza. L'altra pronuncia il timore che ciò non avvenga, manifestando pessimismo e ribellione ${ }^{6}$. Diverso sembra anche il tipo di relazione spaziotemporale con la realtà che le visioni utopiche e distopiche adottano. L'utopia è una proiezione di un "non-luogo", una realtà irrealizzabile. Propone alla società moderna un ideale da raggiungere, offrendo processi alternativi per rispondere a una realtà storica avvertita come dolorosa. La distopia, al contrario, diventa un tipo di proseguimento del processo storico. Partendo dall'evoluzione di tendenze e condizioni contemporanee negative, crea un possibile futuro mondo distorto, "un luogo cattivo", cercando di mettere in evidenza i pericoli a cui si andrà incontro se si continuerà la via attualmente intrapresa?. Come sostiene Romolo Runcini, "se utopia è vita, distopia è morte". Entrambe però, celano un auspicio di fondo che si possa evitare la catastrofe e migliorare il mondo. Le distopie "speranzose" - verrebbe da dire quelle di stampo atwoodiano - si allontano dalla distopia pura e la tradiscono. Vagheggiando, poco prima della fine della storia, un grembo miracolosamente fertile, un percorso di salvezza, sentono il richiamo dell'utopia ${ }^{10}$.

4. Molti critici tendono a separare la distopia dell'antiutopia, assegnando alla seconda il ruolo di rappresentare il rovesciamento ironico della "società perfetta" $\mathrm{e}$ la denuncia dei fallimenti dei progetti utopici di miglioramento del mondo (MuzzIoLI Francesco, Scritture della catastrofe, Roma, Meltemi, 2007, p. 21).

5. SzACKI Jerzy, Spotkania zutopią, Warszawa, Iskry, 1980, p. 183.

6. ANia Gillian, "Apocalypse and Dystopia in Contemporary Italian Writing", in Ania Gillian, Hallamore-Caesar Ann (eds.), Trends in contemporary Italian narrative, Newcastle, Cambridge Scholars Publishing, pp. 155-181: p. 157 (http://usir.salford. ac.uk/id/eprint/1782/).

7. Arrigo Colombo, Baldini Enzo (a cura di), Utopia e Distopia, Firenze, Dedalo, 1993, pp. 11-12.

8. Runcini, Romolo, “Distopia”, IF, Anno III, n. 7, luglio 2011, p. 5.

9. Muzziol, Francesco, Scritture della catastrofe, cit., p. 148.

10. Secondo Ania, la distopia spesso provoca un'apocalisse (nel senso dell'ultimo cataclisma) che conduce all'utopia finale. Partendo dal simbolismo delle apocalissi culturali, dall'antica Roma in poi, che avevano una connotazione positiva, contemplando la pars destruens come fase catartica, necessaria alla rifondazione di un mondo futuro sulle rovine del precedente, le distopie speranzose seguono il modello distopia $>$ apocalisse>utopia ("Apocalypse and Dystopia in Contemporary Italian Writing", cit. p. 157). Utilizzando la classificazione del "quadrato semiotico" di Greimas, proposta 
Secondo Slavoj Žižek, viviamo alla fine dei tempi ${ }^{11}$. La realtà sembra assomigliare sempre più a una distopia, e fenomeni come il ritorno nelle classifiche di vendita di 1984 di George Orwell, del Mondo nuovo di Aldous Huxley, e del Racconto dell'ancella di Margaret Atwood, rilanciato dalla recente serie televisiva, sembrano confermare questa tendenza. Anche la literary fiction ha abbracciato con entusiasmo le distopie: Il Cerchio (2013) di Dave Eggers si concentra sul problema della crescente capacità di influenza dei social media sulla società, La ferrovia sotterranea (2016) di Colson Whitehead unisce ucronia, steampunk e distopia per raccontare lo schiavismo, Non lasciarmi (2005) di Kazuo Ishiguro riflette sulla clonazione umana a fini medici, mentre Zero K (2016) di Don DeLillo immagina un mondo in cui si sconfigge la morte usando le tecniche di criogenesi.

La letteratura italiana contemporanea, invece, ha sempre dimostrato di non essere incline a coltivare $i$ vari generi di fantascienza. Le pur notevoli opere create da alcuni maestri del Novecento, come Landolfi, Buzzati e Ortese, che non hanno fatto del fantastico il loro unico campo di scrittura, si limitano a costituire episodi autonomi.

Un cambiamento radicale avviene tuttavia nella seconda metà del ventesimo secolo, quando i generi fantascientifici cominciano a essere usati per mettere in rilievo ideali politici, come per sottolineare la funzione sociale della letteratura. A partire degli anni Sessanta la contemplazione della catastrofe diventa per molti intellettuali italiani un'ottima occasione per interrogarsi sulle basi economico-sociali e ideologico-culturali della società in cui vivono ${ }^{12}$, di conseguenza, il romanzo apocalittico del dopoguerra appare come il principale erede della grande tradizione delle distopie filosofiche e letterarie e acquista in Italia una notevole popolarità.

Dopo Pier Paolo Pasolini, come afferma Florian Mussgnug, sono Guido Morselli (Dissipatio H.G., 1977), Antonio Porta (Il re del magazzino, 1978) e Paolo Volponi (Il pianeta irritabile, 1978), gli autori dei testi chiave per lo sviluppo del genere distopico italiano, $i$ quali mettono in discussione i fondamenti ideologici e socio-economici delle loro società turbolente, e propongono modelli alternativi. Il romanzo post-apocalittico di Morselli, che riprende esplicitamente la tradizione del trattato filosofico, racconta una storia di intenso isolamento personale. L'ultimo rappresentante dell'umanità, misteriosamente scomparsa dalla terra, scopre che "uno degli scherzi dell'antropocentrismo" è di percepire

da Francesco Muzzioli, questo sottogenere della distopia deve essere descritto con il nome della non-distopia (Scritture della catastrofe, cit. p. 21).

11. Zizek Slavoj, Vivere alla fine dei tempi, Milano, Ponte alle Grazie, 2011.

12. Mussgnug Florian, "Finire il mondo: Per un'analisi del romanzo apocalittico italiano degli anni settanta", Contemporanea, n. 1, 2003, p. 21. 
l'estinzione del genere umano come la fine del mondo. Altri testi apocalittici includono La distruæione (1970) di Dante Virigili, Il giorno del giudizio (1977) di Salvatore Satta, o Il superstite (1978) di Carlo Cassola.

La preoccupazione per lo stato della società contemporanea e per il suo futuro è anche visibile nella letteratura italiana scritta all'alba del terzo millennio. Se si volge lo sguardo alla produzione letteraria del Xxi secolo, non può sfuggire l'enorme quantità di opere d'impostazione fantascientifica e distopica uscite negli ultimi vent'anni: difatti, sono ormai molti gli autori, alcuni piuttosto noti e premiati, che si ergono a esponenti di un genere che anche in Italia ha trovato una tradizione di una certa importanza. Nonostante diverse ambientazioni, stili di scrittura e tipi di narrazioni, tutti gli scrittori usano il filone distopico per esprimere la crescente preoccupazione per lo stato attuale della società e per il suo futuro. Molti, affermando il dovere morale della letteratura ${ }^{13}$, scrivono per strappare il lettore all'apatia, per reagire prima che sia troppo tardi.

Come nota Enzo Baldini, il panorama degli universi distopici del nuovo millennio non è omogeneo e presenta molti aspetti diversi. I contesti si spostano, gli scenari si riconfigurano a seconda dell'emergenza di turno, ma uno dei motivi distopici à la page, che influenza molteplici aspetti dell'opera letteraria odierna, è il tema delle conseguenze dei cambiamenti climatici. In questo contesto appare il romanzo Sirene ${ }^{14}$ di Laura Pugno, una fiaba futuristica che descrive la rovina irreversibile dell'ecosistema che costringe una società a vivere underwater. Come la maggioranza delle narrazioni distopiche contemporanee, il romanzo non racconta la distruzione mentre avviene, bensì colloca gli eventi in un tempo già a catastrofe avvenuta, e rappresenta le conseguenze del disastro, provocato forse dagli errori della scienza o dall'uso spropositato delle risorse naturali. Ci troviamo in un futuro non specificato, ma molto possibile, in una realtà segnata dalle violenze della catastrofe ambientale, in cui l'atmosfera terrestre si è trasformata al punto da rendere l'esposizione alla luce del sole, non più filtrata dallo strato di ozono, quasi mortale. L’umanità, decimata da una contagiosa neoplasia epidermica, si trasferisce in città sottomarine o si rassegna a morire

13. Nel 1965 Elsa Morante pubblica Per e contro la bomba atomica in cui scrive del dovere morale della letteratura di confrontarsi con la minaccia atomica. Secondo la Morante, lo scrittore non deve chiudere gli occhi davanti all'imminenza della fine del mondo, le sue opere devono opporsi all'apocalisse, avvertire del pericolo immediato e totale che ci circonda ("Pro e contro la bomba atomica", Europa Letteraria, VI, n. 34, marzo 1965. Oggi in Morante Elsa, Pro o contro la bomba atomica e altri scritti, Milano, Adelphi, 1987, pp. 95-117).

14. Pugno Laura, Sirene, Venezia, Marsilio, 2007. 
all'aperto, in lazzaretti sulle spiagge. Gruppo privilegiato, i gerarchi della yakuza, nascosti nelle profondità dell'oceano, godono di atroci vantaggi, come quello di utilizzare i corpi delle sirene, scoperte dagli scienziati vent'anni prima ed ora allevate in apposite vasche e adoperate come bestie da carne o usate come schiave sessuali nei bordelli per gli oligarchi.

Una realtà in cui l'uso di sostanze stupefacenti diventa normale, l'eutanasia si applica con una certa regolarità e il suicidio viene percepito come una pratica lecita anche agli occhi dei cattolici' ${ }^{15}$, è un mondo che "non poteva essere salvato" ${ }^{16}$. Il sentore di decomposizione che pervade ogni pagina del romanzo viene rafforzato dal linguaggio austero, privo di ogni forma di adornamento stilistico ed espressività. L'autrice non offre soluzioni di ripiego, né un ottimistico rovesciamento dello sfascio iniziale, come nel caso delle bopeful dystopias. L'unica speranza che la razza umana sopravviva risiede nella possibilità dell'ibridazione con l'essere mitico-acquatico, rappresentata nel finale ambiguo del romanzo.

La catastrofe ecologica diventa il punto di partenza anche di Bambini Bonsai ${ }^{17}$, scritto da Paolo Zanotti:

Ti racconterò i tempi della pioggia, a te che li hai conosciuti solo a stento, nella tua infanzia intermittente di bambina prigioniera. Evocherò per te l'attimo sospeso in cui, dopo mesi e mesi di calura rognosa, apocalittica, omicida, l'afa raggiungeva il suo picco, il tempo era immobile, il corpo si scioglieva: il big bang era vicino, lo si poteva toccare ${ }^{18}$.

Il romanzo di Zanotti racconta la storia di un mondo stravolto dai cambiamenti climatici, dove il caldo quasi mortale si alterna a diluvi epici. L'azione è ambientata in una Genova franata e compressa, in un futuro non troppo lontano dove tutti gli animali sono scomparsi ${ }^{19}$ e tutte le isole del Pacifico sono sommerse da un mare che sembra un mostro di plastica. I bambini, protagonisti principali della storia, approfittando della "vacanza" degli adulti robotizzati,

15. Serkowska Hanna, "Le donne muoiono di Anna Banti (1951) e Sirene di Laura Pugno (2007): un'evoluzione dell'antiutopia femminista", in Dupré Natalie, JANSEN Monica, Jurišić Srecko, Lanslots Inge (a cura di) Narrazioni della crisi, Firenze, Franco Cesati Editore, 2016, pp. 35-43.

16. Pugno Laura, Sirene, cit., p. 35.

17. Zanotti Paolo, Bambini Bonsai, Milano, Ponte alle Grazie, 2010.

18. Ibid., p. 2.

19. Al contrario delle apocalissi di Volponi, Morselli e Cassola, dove alla catastrofe segue la scomparsa degli umani, l'uomo scompare perché se lo è meritato. L'enfasi cade sulle conseguenze del nostro abitare di questo pianeta. 
rifugiati in letargo nelle loro stanze, si avventurano liberi per le strade del mondo soffocante, alieno e spaventoso.

Il motivo del passaggio all'età adulta nella realtà distopica può essere ritrovato anche nell'ultimo romanzo di Niccolò Ammaniti. In $A n n a^{20}$, l'autore immagina un futuro inquietante dopo la diffusione inarrestabile dell'epidemia causata da un virus, diffuso partendo dal Belgio, che provoca la morte di tutti gli adulti. Solo $i$ bambini sopravvivono, anche se condannati a morte, dato che il virus rimane in forma latente fino a quando pure loro, crescendo, lo risvegliano.

$\mathrm{Al}$ di là dell'evoluzione dell'individuo, una catastrofe può dare anche un impulso a creare un nuovo sistema di organizzazione sociale, che diventa l'argomento principale dell'opera distopica nella sua forma più pura ${ }^{21}$. Nel 2015 sul mercato editoriale italiano appare Metropoli22, di Massimiliano Santarossa, che mette in discussione la ritirata della variante orwelliana nella letteratura distopica contemporanea. Il romanzo narra la vita di un uomo perso in un futuro vicinissimo, l'Anno del Signore Duemilatrentacinque, che raggiunge Metropoli per salvarsi dalla distruzione del mondo, piombato in un irreversibile dissesto ambientale, economico e sociale. Una volta entrato, deve affrontare la quarantena, un periodo di adattamento al nuovo macrocosmo. Attraverso gli occhi del protagonista, l'internato numero 5.937.178, viviamo la quotidianità delle viscere di un'enorme città-stato, popolata da milioni di persone schiacciate dal cielo basso e piovoso su una terra annerita, dominata da leggi che regolano ogni istante dell'esistenza. Assistiamo a metodi disumani, volti alla rieducazione degli abitanti, che vengono ridotti ad automi e la cui esistenza è asservita alla produttività: "Ogni internato ha come compito la continuazione della propria vita in funzione della vita della città. Per questo ogni internato avrà cibo, alloggio, protezione, lavoro [...] Metropoli chiede questo: dovete vivere"23. Anche l'alimentazione viene controllata e assoggettata al rendimento della capacità lavorativa: "Obbligo degli internati è assumere duemilaquattrocento calorie quotidiane, così da ottenere la massima efficienza e la maggiore capacità produttiva"24.

20. Ammaniti Niccolò, Anna, Torino, Einaudi, 2015.

21. Secondo la classificazione di Antoni Smuszkiewicz, la distopia rappresenta la visione pessimistica del futuro, basata sull'atteggiamento critico verso la realtà contemporanea e il suo corso di evoluzione. L'opera distopica deve ritrarre panorama più ampio della realtà futura e raffigurare abbozzo del sistema dell'organizzazione sociale ("W kręgu współczesnej utopii”, Fantastyka, n. 6 (33), giugno 1985, p. 60).

22. Santarossa Massimiliano, Metropoli, Milano, Baldini e Castoldi, 2015.

23. Ibid., p. 20.

24. Ibid., p. 30. 
La produzione del cibo, la gelatina distribuita come pasto quotidiano presuppone la macellazione degli uomini, dato che "niente è più nutriente dell'uomo per l'uomo" 25 . Le nuove forme di lavoro, nuovi metodi di organizzazione sociale e di controllo totale, tutto in nome e per la salvezza della città divenuta essa stessa un nuovo Dio, l'ultimo baluardo di asfalto, cemento e metallo di un mondo post-apocalittico.

Decisamente, un tono più che orwelliano prevale anche in Di ferro e d'acciaio ${ }^{26}$, l'ultimo romanzo di Laura Pariani. La scrittrice teletrasporta il lettore in un Nord Italia del futuro, arido e polveroso, dove una mono-Città sopravvive grazie alla tecnologia e al controllo e tutto viene subordinato al modello meccanico di esistenza. Ogni forma di attaccamento diventa proibita e tutti i sentimenti "nocivi" come l'amor materno, la paura, la nostalgia o il desiderio della libertà vengono costantemente monitorati e controllati dai tentacoli di un Grande Fratello ipertecnologico e tecnocratico.

L'elemento tecnologico è sempre molto presente nelle visioni distopiche, in quanto trasforma il mondo, l'uomo, la percezione della realtà e l'evoluzione futura del genere umano. La trasformazione in atto obbliga tutti a riflettere sul fenomeno della pervasività e diffusione degli strumenti tecnologici e degli effetti della tecnologia. La tendenza esasperata all'abuso della tecnologia si concretizza nell'immaginario distopico anche in ambito non italiano, in cui l'inorganico si fa organico grazie allibridazione, come nell'episodio Torna da me della serie televisiva britannica Black Mirror, alla clonazione del succitato Non lasciarmi di Ishiguro e all'ingegneria genetica in Il nuovo mondo di Huxley.

Nel 2014 sul mercato editoriale italiano appare il romanzo La ragaz:a meccanica ${ }^{27}$, vincitore di molti premi letterari, scritto da Paolo Bacigalupi, uno scrittore statunitense di origini italiane. La storia, con evidenti echi cyberpunk, ambientata in un mondo futuro sconvolto da una crisi energetica a seguito dell'esaurimento del petrolio, racconta di un'umanità impegnata nella lotta per la sopravvivenza contro l'innalzamento del livello dei mari che ha devastato il pianeta e distrutto tutte le città costiere. A causa dell'attività di alcune potenti multinazionali, note come "compagnie caloriche", le riserve alimentari mondiali vengono distrutte e il mercato si ritrova costretto all'acquisto di organismi geneticamente modificati. Le disinvolte manipolazioni genetiche portano alla

\section{Ibid.}

26. Pariani Laura, Di ferro e d'acciaio, Milano, NN Editore, 2018.

27. Bacigalupi Paolo, La ragazza meccanica, Terni, Multiplayer Edizioni, 2014. La prima edizione viene pubblicata originale negli Stati Uniti nel 2009. 
diffusione di malattie e piaghe letali che mettono in pericolo la sopravvivenza stessa dell'umanità. Una delle poche nazioni che sono riuscite a ribellarsi all'egemonia delle multinazionali, dopo aver chiuso le frontiere e imposto severi controlli sulle importazioni, è la Tailandia. In questo contesto appare Emiko, la "ragazza meccanica" del titolo del romanzo, frutto della bioingegneria giapponese, creata e programmata per servire come geisha. Le Neo Persone, gli esseri costruiti in laboratori, diventano schiavi, soldati e giocattoli degli esseri umani ${ }^{28}$.

È la storia distopica di un'umanità investita da una crisi senza precedenti che minaccia di distruggerla, in cui non troviamo nessun punto di salvezza. È un mondo tutto nuovo in cui in mezzo alle tante malvagità degli uomini quella di un androide risulta essere la figura più umana. La bioetica, i cibi transgenici, i cambiamenti climatici e l'esaurimento delle risorse petrolifere sono questioni molto attuali che rivelano il potenziale distruttivo sia dell'ambiente naturale che della stessa vita umana, insieme all'enorme arsenale tecnico di cui disponiamo. Come afferma Konrad Lorenz, il possesso di tali strumenti è andato molto oltre la nostra capacità collettiva di controllarli e predisporli a un uso benefico e pacifico $^{29}$. E su questo, nell'epoca postumana in cui governano i Big Data, gli algoritmi e la AI (Artificial Intelligence), riflettono gli autori delle distopie che stiamo analizzando. Come sostiene Yuval Noah Harrari, quando la tecnologia ci permetterà di reingegnerizzare le menti umane, l'Homo sapiens scomparirà ${ }^{30}$. Il robot sostituirà l'uomo vitruviano ${ }^{31}$ e la storia umana arriverà alla sua conclusione, dando origine al regno del postumano.

Oggigiorno lo sviluppo tecnologico viene accompagnato dal progresso delle scienze, tra cui un posto di prestigio spetta alla scienza medica. Tuttavia il raddoppiamento dell'aspettativa di vita, conseguita nel corso del xx secolo grazie ai progressi della medicina e dell'igiene, invece di dar luogo a manifestazioni di ottimismo sfrenato, porta all'invecchiamento della popolazione, che diventa un altro motivo della letteratura distopica. Fra gli autori italiani che notano le conseguenze e le

28. Il motivo della costruzione delle robot-schiave costruite per soddisfare ogni piacere e perversione maschile appare nel romanzo Virus Ginoide (1996), scritto da Richard CALDER. Le androidi, dotate di intelligenza artificiale, hanno però il difetto di infettare e modificare il DNA umano.

29. Lorenz Konrad, Das sogenannte Böse: zur Naturgeschichte der Aggression, Vienna, 1963 (tr. it. L'aggressività, Milano, 1969), cap. XIII e XIV.

30. Harrari Yuval Noah, Homo Deus: Breve storia del futuro, cit., p. 69.

31. HAввick Victor, Robot in the style of Leonardo's Vitruvian Man (in BRAidotTi Rosi, Il postumano, La vita oltre l'individuo, oltre la specie, oltre la morte, Roma, Derive Approdi, 2014, p. 186). 
minacce dell'ageismo ${ }^{32}$, nell'epoca orientata alla produttività e ossessionata dal culto della giovinezza, troviamo Lidia Ravera, autrice del romanzo distopico Gli scadut $^{33}$, che immagina uno scenario aberrante, futuristico, ma intriso di immagini stranamente realistiche. Nell'Italia di un futuro prossimo, con il pretesto del "ritorno alla natura" e di una vita più conforme alle leggi dell'evoluzione, il Partito Unico obbliga le persone, al compimento del sessantesimo anno d'età, a ritirarsi in un mondo parallelo, estraneo alla vita attiva. A trent'anni si è protagonisti della propria vita, a sessanta si "scade" e si viene allontanati dalla scena sociale, familiare e lavorativa. Si viene "rottamati", collocati fuori dalla vita pubblica, in una sorta di riposo a tempo illimitato per fare posto al giovane che avanza. Il livello di decadenza, di inadeguatezza qualitativa, è un marchio automatico che trasforma le persone da elementi utili per la società a scarti da accantonare.

Nella letteratura distopica italiana degli ultimi vent'anni, accanto a numerose opere che riflettono sulla relazione tra l'uomo e il suo ambiente, o sul ruolo della tecnologia e scienza, troviamo anche un'analisi sulle future relazioni fra italiani e stranieri. Il tema degli immigrati e i tentativi di impedire le loro ondate è attualmente uno dei problemi più allarmanti e impellenti ${ }^{34}$. La Roma orientalizzata, invasa dai cinesi come conseguenza del cambiamento climatico, ritratta da Tommaso Pincio nel romanzo Cinacitta ${ }^{35}$ è forse il caso letterario e critico di maggior interesse.

Dopo un anno senza inverno, Roma diventa un luogo immerso nel caldo d'inferno, dove esplodono le macchine, interi quartieri vengono abbandonati, e a impadronirsi del centro della città sono masse di cinesi. Sono gli unici che riescono a trarre profitto anche da una crisi economica. "Peggio dell'apocalisse", commenta uno dei protagonisti del romanzo. Il fantasioso Milano ultima fermata ${ }^{36} \mathrm{di}$ Simone Farè, che ritrae una Milano isolata dal resto del paese e dagli stranieri,

32. Il termine è coniato da Robert N. Butler per descrivere la discriminazione della società verso $\mathrm{i}$ più anziani.

33. Ravera Lidia, Gli scaduti, Milano, Bompiani, 2015. Il problema della posizione degli anziani nella società viene presentato come fulcro narrativo anche nel racconto di D'Eramo Luce, Una proposta risolutiva (1988), in Tutti i racconti, a cura di C. Bello Minciacchi, Roma, Elliot, 2013.

34. Come sostiene Comberiati, l'analisi dei testi Io, polpastrello 5.423 della brasiliana Christina De Caldas Brito, L'ultimo immigrato dell'argentino Miguel Angel Garcia, e I lupi della notte dell'algerino Amor Dekhis mostra la possibile connessione tra letteratura distopica e tema della migrazione (Comberiati Daniele, Distopie identitarie/ Antiutopie diasporiche. Immaginare il futuro all'interno della letteratura migrante, Bologna, Clueb, 2012, p. 86).

35. Pincio Tommaso, Cinacittà, Torino, Einaudi, 2008.

36. FARÈ Simone, Milano ultima fermata, Milano, Cabila, 2009. 
o L'uomo verticale ${ }^{37}$ di Davide Longo, che immagina un piccolo paese assediato dai nuovi barbari, rappresentano ulteriori tappe di evoluzione dello stesso tema.

Particolare attenzione deve essere rivolta anche al romanzo, molto orwelliano nell'impostazione, Il condominio di Via della Notte $e^{38}$ di Maria Attanasio. A Nordia, nome che allude all'idea della Lega Nord di creare la regione indipendente della Padania, futuristica città che non esiste sulle mappe, vigilanza è la parola d'ordine per realizzare un sogno di perfezione collettiva, fondato su un decalogo ferreo che presuppone disciplina e un consenso sociale estremo, basato sull'intolleranza. Gli immigrati possono semmai vivere ai bordi della città, mentre gli abitanti nativi, subordinati alla dittatura e al controllo, non sono in grado di distinguere la costrizione dalla libertà.

Tutte le opere esaminate, nonostante le differenze formali e stilistiche, ritraggono l'umanità all'alba del terzo millennio. Le immagini del futuro, sconvolto dalla crisi, segnato dalle violenze della catastrofe ambientale o tecnologica, fanno paura e terrorizzano perché, a ben guardare, sono molto simili al nostro presente. Anche se prende quota la variante wellsiana della distopia, in cui la catastrofe che avviene o minaccia l'uomo è comunque man-made, la distopia di stampo orwelliano, secondo cui esisterebbe un potere, una forza o un'organizzazione superiore che manipola l'uomo, trova maggiori fautori nel mercato editoriale italiano, con la riserva che oggi cambia quel che si intende per "uomo".

Esaminato il corpus delle distopie dei nostri anni, sembra che uno dei loro motivi più salienti sia la necessità di proteggere il genere umano dai rischi connessi al suo proprio potere e alla sua audacia, in una parola, alla bybris umana, manifesta in molti campi della nostra attività. Diversamente da molte odierne distopie cosiddette "della speranza", le opere qui esaminate non offrono quasi mai soluzioni di ripiego, non riservano alcun rassicurante spiraglio utopico in extremis. Quando è l'homo sapiens a rappresentare l'elemento non migliorabile, la speranza sembra davvero agli sgoccioli. Nessuna meraviglia, considerato che nel nostro antropocene ormai allo stremo il mondo intero, diventato un unico ecosistema, è dominato in modo irreversibile e indivisibile da un'unica specie autodistruttiva e pericolosa per il pianeta. L'uomo.

Aleksandra Pogonska-BaranowsKa Uniwersytet Warszawski

37. Longo Davide, L'uomo verticale, Roma, Fandango Libri, 2010.

38. Attanasio Maria, Il condominio di Via della Notte, Palermo, Sellerio, 2013. 
